merely to say that in each of these cases I found the ganglia at the base of the brain, and more especially the corpora striata, notably hyperæmic; and, proceeding by a method different from that of Dr. Dickinson, I also found occlusions, though not to the same extent in each of them, in the small vessels of these parts; the occlusions being partly fibrinous plugs, and partly composed of rounded concretions, apparently of an albuminoid nature, such as are figured in the righthand part of the illustration on page II of the Transactions of the Pathogical Society for I869. In Dr. Wilson Fox's case, where delirium supervened, there was also a minute capillary injection of many parts of the surface of the brain; and in these regions I also found numerous occlusions of minute vessels.

My observations were made in the latter case by separating the convolutions whilst the brain was in the fresh state, cutting with a sharppointed scissors through and all round a portion of the pia mater adhering to one side, and removing this, with as little traction or disturbance as possible, to a microscope-slip, where it was immersed in a quarter per cent. solution of bichromate of potash. The portion of pia mater was put into this thin fluid for preliminary examination, in order to prevent shrivelling; and afterwards, if it were to be preserved, glycerine was allowed to run in and replace the bichromate solution without disturbing the specimen. In examining the corpora striata and other internal parts of the brain, after section of the part, a distended vessel was dissected out by means of a needle, whilst its surrounding branches were cut across with a sharp-pointed scissors whilst they were still imbedded in the brain-substance. The vessel and its branches were then removed as before, with as little disturbance as possible, to a weak bichronıate solution. In this way, the contents of the vessels and their branches could be fully examined, and I was able to detect the vascular occlusions which I believe to be the principal cause of the minute injections of the parts, but which Dr. Dickinson seems not to have found in his examination of sections of the hardened organs. In future observations, the two methods should be combined; that is, with different parts of the same brain. The examination of the vessels by removing them from the recent brain is good as a preliminary measure best calculated to reveal the fact of the existence and the nature of occlusions; but, in order to study the effects produced by them, we must proceed, as Dr. Dickinson has done, to harden the nervous organs and subsequently examine sections.

[To be continued.]

THE EMBOLIC THEORY OF CHOREA.

By THOMAS STRETCH DOWSE, M.D., F.R.C.P.E., Physician-Superintendent of the Central London Sick Asylum, Highgate, etc.

MY experience in that acute angle of the plane of nervous diseases called chorea has not been so extensive as in the remaining superficies. Yet I think I have seen sufficient of the etiology, clinical features, and pathology of this disease and its kindred congeners to venture a few remarks on this very important question.

I admit, on commencing, that, of the four cases where I have had an opportunity to examine the cerebral vessels, no embola were detected, but thrombi of the vessels of the grey matter of the convolutions of the postparietal and occipital lobes were abundant, as well as perivascular spaces and degenerated nerve-cells. Of this I am sure, that, so long as we constantly write and talk of embolism of cerebral vessels to the exclusion of thrombosis, so long shall we be in error and fall short of a most important and unvarying phenomenon.

It is to the vascular system and the stability and integrity of the fluids in circulation through the body, that we have to look for the induction of primary changes in the nerves and nervous centres. It is the circulation of the blood-governing, as compared with the due formation of the blood-governed, which we have to take into consideration; and, moreover, it is to the due correlation of these that we have to look for a healthy standard. I ask the questions : Is an atheromatous vessel due to blood-change, or to defect in the governing power which regulates its nutrition? Is a cerebral glioma or fibroma of the dura mater due to blood-change or to defect in the governing power which regulates the due nutrition of those parts? Is an ovarian growth or uterine cancer due to primary blood-change or to defect in the governing power which regulates nutrition?

The probable answer will be, that it is sometimes the one and sometimes the other. I cannot meet the question without committing myself to mere conjecture, which I should be very sorry to do. Still, it would be a not uncommon error, in these metaphysical days of advanced pathological research, did I venture to state that, in the habit of body or constitution known as the choreic, we have a family history, a dia- thesis, a blood-condition, a nervous state; in fact, a type where the governing power, the blood, is easily aborted and becomes, in great measure, governed by nervous agency rather than being itself the governing agent, and it is thus that disease commences.

It might be chorea, pericarditis, valvular or atheromatous changes, acute rheumatism, epilepsy, mental derangement, the various forms of skin-disease, mere albuminuria, or desquamative nephritis, age and circumstances modifying results as influenced by hereditary tendency.

I do not think it would be an easy task to say whether the blood or the nervous system was the factor of thrombosis in the following cases which I find in my note-book.

A woman, aged 50 , was sitting at tea in her usual health during a hot sultry summer afternoon; she was suddenly startled by a loud peal of thunder, and immediately she was seized with intense pain at the uppermost part of the thigh. When she came under my care, the limb was of immense size, and a plugging of the iliac and femoral veins was diagnosed and verified by post mortem examination.

A woman, aged 36 , was standing on shore and saw her son drowned in a gale at sea. She suffered from temporary mental aberration, which was succeeded by general incoordinate muscular movements of the face and extremities, with opispleurosthotonos. Here, we had multiple thrombosis of the grey matter of the brain and spinal cord. She recovered with some ataxy of the lower limbs.

A man had been suffering from repeated attacks of erysipelas, but had recovered. There was ankylosis of the joint of the index-finger, which was amputated. In less than three hours, his temperature rose to I04 deg., the respirations to 60 , and he died from what is called shock within twenty-four hours of the removal of the finger. At the post morlem examination, the heart was found to be empty, but the vessels of the brain and spinal cord were plugged with thrombi.

I have cited these cases, as I could many others, to show (but not to prove) that, in certain conditions of the blood and nervous system, thrombosis is not so uncommon. We hear a great deal of vascular spasm in the various forms of epilepsy, true enough; but I believe ramuscular venous thrombosis has been overlooked. I maintain that no one can give a rational explanation as to the pathology of chorea who does not first make himself acquainted with the causation of the many forms of involuntary. incoordinate movements which are to be found in well known organic changes of the nervous system. Let it be granted that, in chorea, we have something unique : movements, in fact, which can scarcely be called tonic or clonic, whose diverse action partakes of flexion and extension of parts supplied by the same nerves at the same moment of time without rhythm, uniformity, or regularity, with or without loss of sensation, but, unless very profound, are certainly, in some measure, under mental influence.

In Dr. Hughlings Jackson's able defence of the embolic theory of chorea, he states : "All movements must depend on nervous discharge. Disintegrated nerve-tissue cannot discharge ; for really the disintegrated matter has ceased to be nerve-tissue. I suppose the excessive movements occurring either in chorea, or epilepsy, or epileptiform seizures, are produced by discharge of grey matter, which, except for great instability from overnutrition (not better nutrition) is healthy."

One can but be in accord with Dr. Jackson, that disintegrated nerve-matter ceases either to generate or conduct nerve-force, yet there are periods of disintegration which cannot be overlooked. The first of these, of the utmost importance, is molecular derangement. It might arise from mere inhibitory action of reflective centres, or from the least possible transudation of serum from overgorged vessels. Nerve-force is essentially vital force, and, as in the inorganic world we have latent force, so much the more have we latent force in the organic. This, I presume, no physiologist is willing to deny. It is in molecular derangement, inducing molecular hyperæsthesia (irritability), that this latent nerve-energy is generated, not from overnutrition.

There is no such thing as overnutrition beyond what is compatible with perfect health. After this nutrition is perverted and degeneration begins, and so surely commences what Dr. Hughlings Jackson calls the positive condition of the motor organs in chorea, the negative follows when disintegration is complete. The questions which arise in reference to the cause, course, and termination of chorea (independent of post mortem microscopic evidence) do not to my mind favour the embolic theory. But one is bound to admit, and many cases have occurred in my own practice where extensive valvular disease has given rise to embola of the left Sylvian artery and its branches, which has been productive of defective speech, right facial palsy, jactatory involuntary movements of the arm and paresis of the leg, without spasm.

One case I remember of extreme interest, and would at first sight support the embolic theory; but the movements were unlike those of chorea, and the area of distribution of nerve-change gave evidence of a more limited destruction than is found in hemichorea. 
The association of chorea with valvular disease of the heart does not support the embolic theory, or, to say the least, does not maintain it ; for the latter, as a rule, does not precede the former, but originates in direct ratio with the persistency of the choreic movements, inasmuch as chorea, acute rheumatism, and valvular disease of the heart are directly associated in the same habit of body and nervous temperament. So far in this respect might it be inferred that embola were productive of chorea, but one cannot push this point of the argument farther. ask the question, Is it possible that the initiative nerve-change or irritability over any given cerebral area, can be maintained after the absolute causative agent has been removed ? or, on the other hand, is it possible that the lesion which gave rise to the abnormally active impulse has so impressed the nerve-matter as to induce in it a property of hebetude which acts in correlation with subjective, attendant, and perverted mental processes? We know that a choreic patient will, when occupied, perform many movements quite co-ordinately which it is impossible for her to do whilst under observation.

The automatic balance of nerve-function is here superseded by emotional hypergenetic agency. Such a condition as this is the outcome not infrequently of what is often termed nervous mimicry, and there can be no doubt that such is frequently the case without what is usually understood as gross pathological change. If pathology do not serve us (and as yet this is perfectly true that it does not) in clearing up by demonstrative proof many vexed questions in reference to the cause of nervous disease. There are, on the other hand, many objective factors at work evidenced in the shape of clinical features, or signs, or symptoms, which, if carefully considered, weighed, and compared, will lead us to a correct and truthful issue.

In the cases of well marked embolic plugging which have come under my observation, the results have been, as before remarked, so unlike chorea, that I am inclined to think the theory untenable; and, judging from my own pathological data, such condition is absolutely negative; and the fact that the middle cerebral artery was found plugged by Dr. Moxon in a case of hemichorea followed by hemiplegia does not by any means invalidate this conclusion ; in fact, it rather goes to prove that chorea was followed (by association) with valvular disease of the heart, which gave rise to the embolon that plugged the Sylvian vessel, and that this plugging gave rise to the hemiplegia.

\section{ON THE DRESSING OF WOUNDS.}

\section{By EDMUND OWEN, F.R.C.S.,}

Assistant-Surgeon to St. Mary's Hospital, and to the Hospital for Sick Children.

MR. RIChard DAvy's clinical remarks on the dressing of wounds, which appeared in the JourNal of December 30 th, 1876 , are as bold as they are well timed. Mr. Davy comes forward as a champion of simplicity and æsthetics in surgical dressing; and, at the present day, when surgeons of the so-called antiseptic school are prone to attribute their success to some special virtue in carbolic acid, or in the way in which they employ it, Mr. Davy's trenchant remarks are of the greatest value.

By their side, I would like to place a few extracts taken from the lectures of a surgeon and teacher of vast and ripe experience. Thus we read in Spence's Lectures on Surgery (vol. i, p. 140, 2nd edition):"Some of the statements advanced in favour of the antiseptic system so ignore the success ohtained by simple dressing and treatment of wounds, or assert such an amount of infallibility as to the curative powers of the special method, as to require notice." Such statements, the author considers, arise from want of experience in, or misrepresentation of, the simple method of treating wounds. Further on, he says : "Novelty is not always progress, and unfortunately many novelties in surgery at the present day seem to consist in departure from simplicity of treatment."

The so-called antiseptic method of treatment is held by some of its practitioners to be all but infallible. Indeed, if such an one hear of a case treated " antiseptically" where the issue was unhappy, he is apt to say that there must have been a flaw somewhere in the intricate process. On the other hand, the patient recovers; there the treatment was carried out in its entirety.

Mr. Davy gives us thirty-two cases of important operations without a death, and without any special antiseptic treatment having been adopted. No disciple of any school, however modern, can show better results.

I will now venture to point out how it is that Mr. Davy has been so successful in his hospital practice. Like practitioners of the so-called antiseptic method, Mr. Davy pays the most careful attention to his patients after operations; he does not leave them (as is often the case in hospital practice) too much to the care of house-surgeons, dressers, and nurses. I speak now with all respect for house-surgeons, but it cannot be denied that they are not always competent to take entire charge of a patient during the critical phases which present themselves in the first few days after an operation. In my experience, there is something for the surgeon to suggest, or to see carried out, each time he sees his patient in the first week, no matter how often he comes. Now, this is just where the antiseptic surgeon, in my opinion, effects so much. $\mathrm{He}$ is continually seeing his patient. Nothing is left to an untrained assistant or to chance. His dresser is a picked man and is specially instructed. Nothing is neglected ; for everything is done in order and under the surgeon's personal and watchful supervision. The time and labour which he gives to his patient meet their due reward.

Ambroise Paré, whom Mr. Davy quotes with much effect, used to say of his patient in simple faith : "I dressed him ; God cured him." But I apprehend that Pare's watchful care did more for his patients than did his "balsame of boyled whelpes and wormes".

Without presuming to adduce statistics from the work of five or six short years of hospital practice, I would, nevertheless, venture to make a few remarks upon the dressing of wounds. I dislike poultices ; sometimes they are applied so hot as to distress the patient considerably. At other times, they are little more than warm. They are always dis. agreeable and unwholesome. Often the wound is allowed to become chilled whilst a fresh poultice is to be applied. Moist warmth can, when necessary, be obtained in many better ways.

Sores, however foul, may be cleansed and kept absolutely sweet by the liberal use of sulphurous acid lotion, which is of far more value than the fashionable carbolic acid lotion. For the treatment of fresh wounds, nothing is better than dry lint : I have left it on a wound after a herniotomy for three weeks, and, at the end of that time, have found the wound represented by a healthy cicatrix.

As Mr. Davy reminds us, the wound is but a single element in the case, and I apprehend that, if a patient be carefully watched and subjected to well considered hygienic influences, Nature will repair it, unless the interference on the part of the surgeon be too officious.

\section{THE USE OF SPONGE-PADS IN EXCISION OF TUMOURS.}

By W. WATSON CAMPBELL, M.D., F.R.C.P.E., Dunse.

In the British Medical JoURnal of October 2 Ist, I876, I observe a note on a case of amputation of the breast, in which it is stated that the wound was healed in seventy-two hours after operation. There is a suspicious reference to continued high pulse and a drop or two of pus, which makes me wish I heard more of this case; for it occurs to me that these are sufficient to give rise to a fear that the result may not have proved what Mr. Maunder expected. $\mathrm{Mr}$. Maunder speaks of pads, and I have sometimes wondered that what are usually employed as such, viz., lint, tow, towels, etc., have not long ere this been superseded by something better-sponges. Short as the time of healing in this case is reported to be, it does not strike me as extraordinary, but merely what might be obtained in a great majority of cases were sponges used as pads. I have been for years in the habit of using sponge-pads in the excision of tumours, and the rule has been union by first intention. There may be one or two points in the method I adopt that are worthy of attention;; and, even if these be not original, I hope I shall be excused for bringing them before the profession.

After excising a tumour, I pare the edges, if necessary, and do not hesitate to remove anything-such as fat-which might interfere with accurate apposition of the flaps and the margins of the wound. Before closing the wound, I wait until every drop of blood has ceased to flow ; for I believe that even slight capillary oozing is sufficient to prevent primary healing, by keeping the internal and opposing surfaces separate. Having carefully applied the sutures, I press a sponge firmly over the place from which the tumour has been removed, so as to force out between the stitches any blood which may have escaped into the cavity during the sewing. I then lay a bit of dry soft linen or bleached cotton-cloth over the place, and over this apply a dry clean sponge of sufficient size to produce, when bound down, such an amount of pressure as will serve to keep the internal surfaces close together and prevent capillary hæmorrhage. The advantage of linen or cotton over lint is, that neither has fibres like those of lint, which cause it to stick into the edges of the wound, and give rise to trouble to the surgeon and uneasiness to the patient when it is removed. The sponge is light, soft, and elastic, and readily adapts itself to such irregularities as there may be in the cavity from which the tumour has been removed. A 